\title{
Modifications to the hand-held Gene Gun: improvements for in vitro Biolistic transfection of organotypic neuronal tissue
}

\author{
John A. O'Brien ${ }^{a}$, Matthew Holt ${ }^{a}$, Garth Whiteside ${ }^{b}$, Sarah C.R. Lummis ${ }^{\text {a,c }}$, \\ Michael H. Hastings ${ }^{\text {a,* }}$ \\ ${ }^{a}$ Neurobiology Division, MRC Laboratory of Molecular Biology, Hills Road, Cambridge CB2 2QH, UK \\ ${ }^{\mathrm{b}}$ University Department of Anaesthesia, Level 4 Addenbrooke's Hospital, Hills Road, Cambridge CB2 2QQ, UK \\ ${ }^{\mathrm{c}}$ Department of Biochemistry, University of Cambridge, Tennis Court Road, Cambridge CB2 1GA, UK
}

Received 20 March 2001; received in revised form 17 July 2001; accepted 7 August 2001

\begin{abstract}
Transfection and subsequent expression of DNA in living neuronal tissue is problematic and no technique has emerged that is completely non-damaging, efficient and reproducible. The Bio-Rad hand-held Gene Gun has overcome some of these problems by exploiting a biolistic method in which small gold particles carrying plasmid DNA are propelled into neurons whilst causing minimal detectable cell damage. In its current configuration, however, the Bio-Rad Gene Gun is optimised for transfecting cells in dispersed cultures, and therefore delivers particles superficially over a relatively wide area. Here we report modifications to the Bio-Rad Gene Gun that both enhance its accuracy by restricting its target area, and increase the depth penetration achieved by gold particles, thereby allowing smaller and deeper tissues to be transfected. These alterations make the modified Gene Gun more applicable for in vitro transfection of organotypic cultures and enhance its potential utility for in vivo gene delivery. Moreover, the modified configuration operates successfully at lower gas pressures, thereby reducing even further the degree of cell damage incurred during transfection. (C) 2001 Elsevier Science B.V. All rights reserved.
\end{abstract}

Keywords: Biolistic gene transfer; Neurons; Fluorescent protein; Dorsal root ganglion; Organotypic cultures

\section{Introduction}

Conventional transfection methods have achieved limited success with neurons (Jiao et al., 1993; Katz et al., 1994; Biewenga et al., 1997; Murphy and Messer, 2001). Biolistic transfection, a technique first introduced as a method of gene transfer into monocotyledonous plants (Klein et al., 1987, 1988) has been applied to neural tissues. This method utilises rapid propulsion of small metal particles to introduce their coating of plasmid DNA into tissue targets. A major advantage is that it is not dependent on specific biochemical features of cell membranes and therefore it has wide applicability across cell and tissue types, and subcellular organelles (Zelenin et al., 1989; Boynton et al., 1993, 1996). An

\footnotetext{
* Corresponding author. Tel.: + 44-1223-402400; fax: + 44-1223402310.

E-mail address: mh105@cam.ac.uk (M.H. Hastings).
}

early configuration, used to transfect neurons in slice culture, holds the specimen in a vacuum chamber, into which microcarrier particles are projected at a very high pressure (Arnold et al., 1994; Jiao et al., 1993; Lo et al., 1994; McAllister et al., 1997; Arnold and Heintz, 1997; Thomas et al., 1998; Tao et al., 1998; Usachev et al., 2000). The hand-held Gene Gun introduced more recently by Bio-Rad provides a second, more convenient configuration for biolistics, and this too has been used to transfect primary neuronal cultures (see Yoshida et al., 1997; Wellmann et al., 1999). The hand-held Gene Gun is, however, optimised to transfect superficial tissues or dispersed cells in culture, propelling microparticles superficially over a wide area. Penetration into deeper tissue is limited. Moreover, the standard configuration induces appreciable tissue damage when used at the higher gas pressures often required for effective transfection (Yoshida et al., 1997; Zhang and Selzer, 2001). The aim of this study was to modify the 
Bio-Rad Gene Gun to enhance its ability to transfect neural tissues in organotypic culture.

\section{Critical parameters in Biolistics}

The Bio-Rad Gene Gun uses a pulse of helium fired through the apparatus to strip gold microcarriers, coated with DNA, from the inner surface of a cartridge constructed of Tefzel tubing. The expanding gas stream propels the particles forwards down the accelerator channel. The pressure of the helium pulse required to strip the microcarriers from the plastic cartridge and project them along the accelerator channel will affect the depth of penetration. It will also affect cell survival, because higher pressures cause a shock wave across cultured neurons, detaching them from the matrix. A second critical feature is the design of the accelerator channel itself. The distal end of the accelerator channel is a flared, cone-shaped outer barrel. As the helium leaves this coned surface, it follows the contour thereby dispersing the gold/DNA particles in a continuing outward motion that defines the extent of the target area (Manufacturer's specifications). The standard configuration of the cone is optimised to project particles over a relatively wide area, and the spacer that is attached distal to the cone barrel determines the radius of this area. This spacer also defines the minimum distance between the cone barrel and the target tissue, and this distance is a third critical feature. There will be a decreased likelihood that gold/DNA particles will penetrate the target if the distance from the barrel to the tissue is too great (Wellmann et al., 1999), but if the distance is too short, there will be an increased risk of tissue damage from the pressure wave.

Performance of the Gene Gun in terms of target area, tissue penetration and tissue damage is determined therefore by the balance between gas pressure, the area of dispersion over the target, and the proximity of the outer barrel to the tissue. The major design feature of the Bio-Rad Gene Gun that affects these factors is the profile of the accelerator channel, which causes the microcarriers to spread outwards from their original $2.0 \mathrm{~mm}$ diameter cartridge to a diameter of up to $1.5 \mathrm{~cm}$ at the target site (Yoshida et al., 1997). The standard configuration compromises the use of the Bio-Rad Gene Gun for accurate, focal transfection of deeper tissues.

\section{Modifications to Bio-Rad Gene Gun design}

The aim was to increase the depth penetration of gold/DNA particle using the same propulsive gas (helium) without damaging the tissue. We therefore redesigned the accelerator channel to form a more compact, focused shot of gold particles. The modification also allowed for a significant reduction of the gas pressure, thereby reducing the likelihood of tissue damage, and also increased the proportion of microparticles propelled from the cartridge surface.

To prevent gold/DNA particles dispersing in an outward motion, the accelerator channel and its distal cone (Fig. 1) were removed and replaced by a modified accelerator channel and external barrel. The new channel and outer barrel were not flared. Instead their walls ran parallel. The modified structure was made of brass (external barrel) and stainless steel (accelerator channel) pressed into a Delrin body. The barrel has baffle holes drilled into it at an angle of $30^{\circ}$. In preliminary trials, prototypes without these baffles were subject to strong

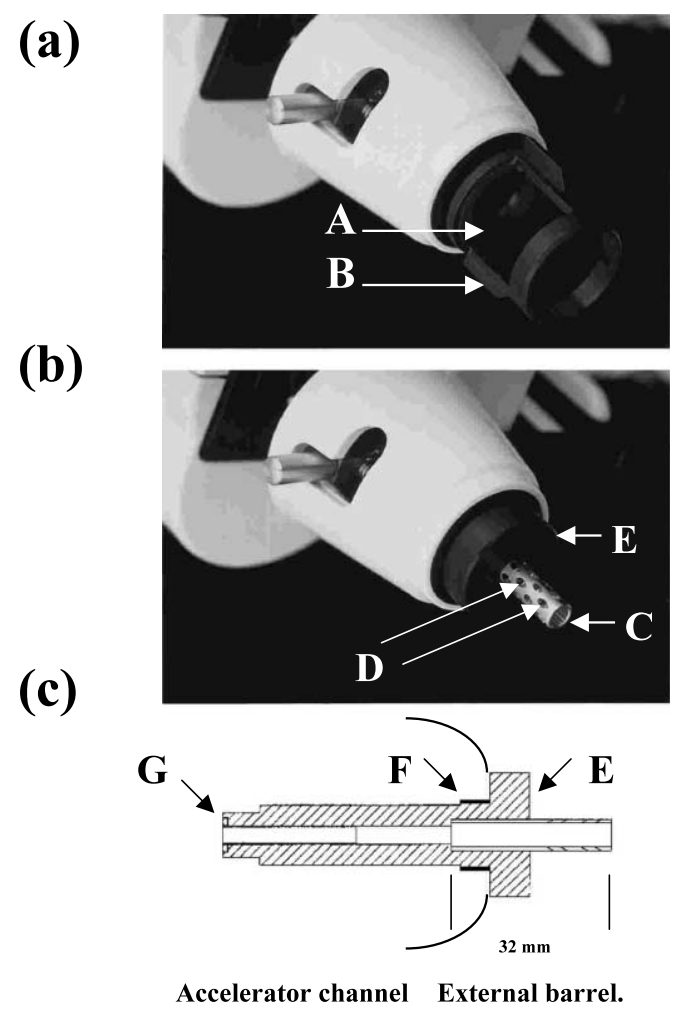

Fig. 1. Modifications to Bio-Rad Gene Gun. (a) Close up view of the Bio-Rad standard Gene Gun displaying the cone shaped surface of the outer part of the accelerator channel (A) and the 'spacer' (B) which defines the minimum distance between target and the accelerator channel cone. (b) In the modified design the 'spacer' has been eliminated, and the cone shaped barrel is replaced with an external barrel of a reduced diameter (C). The external barrel has 20 baffle holes of $1.5 \mathrm{~mm}$ diameter drilled into it (D), each at an angle of $30^{\circ}$ to the long axis of the barrel. A Delrin body (E) carries the modified barrel, securing it to the Gene Gun housing. (c) Schematic cross-sectional view of the modified accelerator channel and external barrel. The external barrel is constructed of brass ( $32 \mathrm{~mm}, 6.3 \mathrm{~mm}$ O.D., 4.5 $\mathrm{mm}$ I.D) and the accelerator channel of stainless steel (3.75 $\mathrm{mm}$ O.D., $2.9 \mathrm{~mm}$ I.D.), both pressed into a $30 \mathrm{~mm}$ Delrin body support (shaded). The whole structure is securely mounted into the Gene Gun via a screw thread (F) located behind a $50 \mathrm{~mm}$ Delrin collar visible as $\mathrm{E}$ in (b) above. A pair of O-rings (G) seal the accelerator channel against the cartridge holder. 
recoil on firing the helium gas. As in the standard apparatus, the accelerator channel fits against the cartridge carrying the gold/DNA microparticles with a tight seal produced by two ' $O$ ' rings. This tight seal directs the flow of helium down the channel towards the outer barrel. The intention was to reduce the spread of microcarriers over the target site, and, by removing the spacer, to allow the apparatus to be placed closer to tissue targets. This is especially useful for small targets such as organotypic tissue slices in vitro, or for surgically exposed tissues in vivo (Zhang and Selzer, 2001).

Bullets used in this study were made of Tefzel tubing (Bio-Rad Laboratories) coated with $1.0 \mu \mathrm{m}$ gold particles carrying plasmid DNA encoding yellow fluorescent

(a)

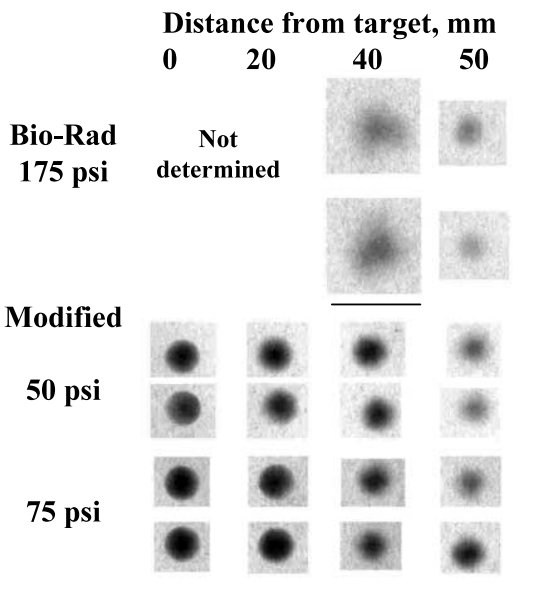

(b)

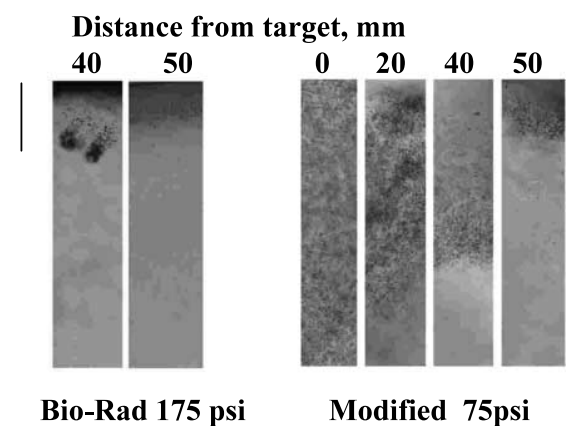

(c)

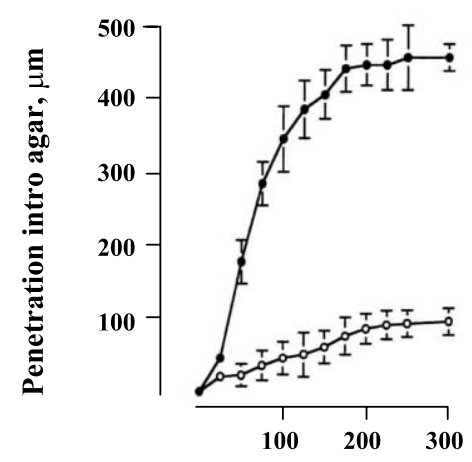

Helium pressure, psi

Fig. 2. protein (CMV-EYFP, Clontech). Gold was used rather than tungsten because the latter tends to acidify culture medium and induce apoptosis of neurons (Sanford et al., 1993). Bullets were made using a modified form of the manufacturer's protocol. Briefly $25 \mathrm{mg}$ gold particles were mixed with $50 \mu \mathrm{g}$ plasmid DNA and spermidine $(50 \mu \mathrm{l}$ of $0.05 \mathrm{M})$. DNA was precipitated by addition of $\mathrm{CaCl}_{2}(50 \mu \mathrm{l}$ of $10 \mathrm{mM})$ and the gold/DNA slurry washed with absolute ethanol and resuspended in ethanol with polyvinylpyrrolidine (PVP, $0.075 \mathrm{mg} / \mathrm{ml}$ ). The slurry was taken up into Tefzel tubing and the ethanol removed immediately as rotation of the tube loading station commenced in order to coat the inner tube surface with gold/DNA particles. In our experience, a delay in removal of the ethanol caused the gold to adhere too strongly to the tubing and coat unevenly. Reduction of the PVP concentration did not overcome this problem (unpublished observations). A flow of nitrogen was used to evaporate the remaining ethanol.

\section{Performance of the modified apparatus on inert targets}

In all cases, for both inert and tissue targets, the standard and modified Gene Guns were used with a 70 $\mu \mathrm{m}$ nylon mesh directly over the barrel. In its absence, tissue damage was markedly increased (unpublished observations). Initial designs included rifling within the accelerator channel and external barrel in order to maintain focus of the shot. This, however, yielded no advantage in performance over a plain barrel and was not incorporated into the final modification (unpublished observations).

In order to define the area of gold delivery, and to

Fig. 2. Performance of standard (Bio-Rad) and modified apparatus on inert targets. (a) Duplicate representative images of dispersion pattern of gold microparticles shot onto filter paper at pressures of 175 (Bio-Rad), or 50 or 75 psi (Modified). For the standard Gene Gun, the spacer was held adjacent to the paper $(40 \mathrm{~mm}$ distance from terminal cone of accelerator channel) or $10 \mathrm{~mm}$ away $(50 \mathrm{~mm}$ total distance). For the modified apparatus, the external barrel was held adjacent to the paper $(0 \mathrm{~mm})$ or at a distance of 20,40 or $50 \mathrm{~mm}$. Note the concentrated target area of the modified apparatus shot at low pressure and at a range of distances from the target surface. The trial $(n=3)$ was conducted independently three times with comparable results. Scale bar $1 \mathrm{~cm}$. (b) Representative images of vertical penetration of gold microparticles into agar. The surface of the agar is at the top of the image. The distance from target indicates the proximity of the standard and modified apparatus, shot at 175 or 75 psi, respectively. Note limited penetration of gold particles shot by the standard apparatus compared to the modification. Scale bar = $100 \mu \mathrm{m}$. (c) Comparison of the effect of pressure on the penetration into agar of microparticles shot by either the standard (open circles) or the modified Gene Gun (closed circles). Data represent mean \pm SEM of three trials at each pressure. The modified apparatus was held $40 \mathrm{~mm}$ from the agar surface; the standard apparatus had the spacer adjacent to the agar surface 
optimise the pressure of helium and the distance from the target, gold/DNA microcarriers were shot at Whatman No. 1 filter papers. The conventional Bio-Rad apparatus fired at 175 psi with the spacer against the paper (i.e. target $40 \mathrm{~mm}$ from the distal cone of the accelerator channel) produced a diffuse area of gold microparticles ca. $1.5 \mathrm{~cm}$ in diameter (Fig. 2a). Moving the spacer away by $1 \mathrm{~cm}$ from the paper greatly attenuated the area of gold visible on the target, presumably due to dispersion of the gold over a greater area. There was no visible deposition of gold microcarriers when the Bio-Rad apparatus was used at a pressure of either 75 or 50 psi (data not shown). In contrast, the modified apparatus was very effective at projecting gold at the target when operated at these lower pressures. With the tip of the external barrel against the target, the modified apparatus produced a very tightly circumscribed deposition of gold when fired at either 75 or 50 psi. The diameter of deposition was approximately 0.5 $\mathrm{cm}$. The focal area of deposition was maintained with increasing distance (up to $50 \mathrm{~mm}$ ) of the external barrel from the target, although at the greatest distance $(50$ $\mathrm{mm}$ ) there appeared to be an attenuation of target coverage, especially at $50 \mathrm{psi}$. The greater intensity of the image of gold particles on the filter paper produced by the modified apparatus likely reflects both the more focused distribution of the gold projectiles, and the greater efficiency with which it stripped gold particles from the Tefzel tube cartridge (see below).

Having established effective parameters of pressure and distance for the modified apparatus, the extent of particle penetration was then assessed by firing at agar plates. The distribution of gold particles in the agar column was determined using a Bio-Rad MRC 600 confocal microsope. At a pressure of 175 psi and held with the spacer against the agar surface (cone therefore $40 \mathrm{~mm}$ from target) or $10 \mathrm{~mm}$ away (cone $50 \mathrm{~mm}$ from target), the Bio-Rad apparatus caused a slight depression to the agar surface and propelled gold carriers into the agar to a maximum depth of around $150 \mu \mathrm{m}$ (Fig. $2 \mathrm{~b})$. With the modified apparatus used at 75 psi and held directly against the agar surface, there was surface damage to a depth of $50 \mu \mathrm{m}$, but gold particles penetrated to a depth of up to $500 \mu \mathrm{m}$. Moreover, the spread of particles through the agar column was relatively uniform. As the tip of the modified apparatus was withdrawn further from the agar surface, there was a progressive decrease in penetration. Nevertheless, when held $40 \mathrm{~mm}$ from the surface and fired at $75 \mathrm{psi}$ the penetration achieved by the modified apparatus was in the region of $300 \mu \mathrm{m}$.

In a systematic analysis of the effect of pressure, both types of apparatus were placed with the cone (standard Bio-Rad) or the external barrel (modification) $40 \mathrm{~mm}$ from the agar surface, and penetration was determined over a range of pressures between 20 and 300 psi.
Regardless of pressure, the standard Bio-Rad apparatus penetrated no further than $100 \mu \mathrm{m}$ (Fig. 2c). In contrast, the modified barrel demonstrated increasing penetration over the range of $20-75$ psi, reaching a mean value of about $440 \pm 55 \mu \mathrm{m}$. At a pressure of $75 \mathrm{psi}$, penetration was in the region of $275 \mu \mathrm{m}$, and visual inspection of the bullets indicated approximately $80 \%$ of the gold particles were ejected with the modified barrel. In contrast, the standard Bio-Rad apparatus did not discharge appreciable amounts of gold when used at this pressure (nor at 100 psi, see below). In addition to its more focused coverage of the target and enhanced penetration, the modification therefore provides the important advantage of allowing the Gene Gun to operate very effectively at low $(<100$ psi) pressures.

\section{Transfection of cultured tissue with the modified apparatus}

Having established that the modifications to the original Bio-Rad apparatus enhance its accuracy, penetration and effective operation at low pressures, its functionality was assessed further by testing the transfection of dispersed cell cultures, organotypic cerebellar slice cultures and spinal dorsal root ganglion (DRG) explants.

Confluent cultures of HeLa or HEK293 cells, grown on $19 \mathrm{~mm}$ glass coverslips, were shot with either the Bio-Rad apparatus at $175,100,75$ or 50 psi or the modified apparatus at 75 or 50 psi. Successful transfection was identified by the expression of an EYFP reporter gene, and the area of cell loss and damage was identified by Hoechst 33342 nuclear stain. Consistent with the observations on inert targets, no transfected cells were observed with the standard Bio-Rad operated at 100,75 or 50 psi (data not shown). When used at 175 psi, the standard Bio-Rad destroyed cells immediately in the centre of the coverslip (Fig. 3a), although cells 1 $\mathrm{mm}$ from the centre appeared healthy. Nevertheless, there was very little transfection of either HeLa or HEK cells in the central $6 \mathrm{~mm}$ diameter area (Fig. 3a, b). In contrast, the modified apparatus used at 50 psi caused little cell damage and the central region of the culture exhibited very high transfection levels. At the higher pressure of $75 \mathrm{psi}$, the modified apparatus did cause cell damage, as evidenced by detachment and apoptotic nuclear profiles. Nevertheless high levels of transfection were observed close to the target centre, peaking at a radius of $3 \mathrm{~mm}$ (Fig. 3b). Beyond this range, the frequency of transfection fell markedly, whereas with the standard Bio-Rad apparatus high levels of transfection were observed in a broad band of cells between 3 and $10 \mathrm{~mm}$ from the target centre. The maximal diameter (mean \pm SEM) of the central area of cell loss was $927 \pm 71 \mu \mathrm{m}$ for the standard apparatus 
(a)

Bio-Rad
175 psi

Modified

50 psi

75 psi
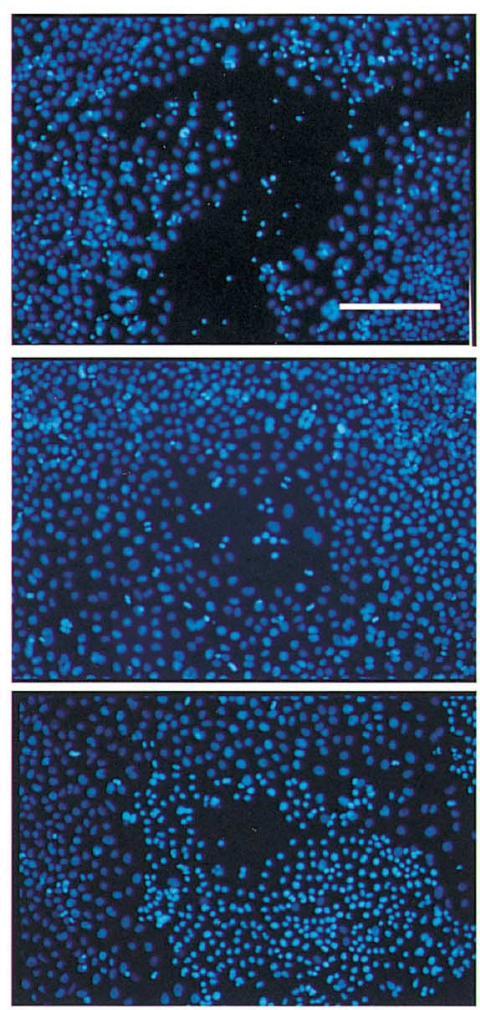

Hoescht

(b)
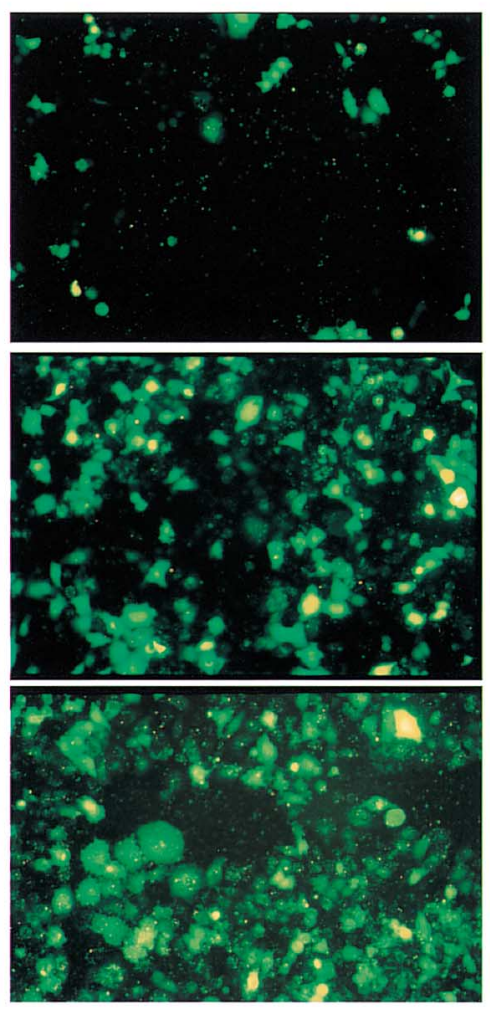

EYFP
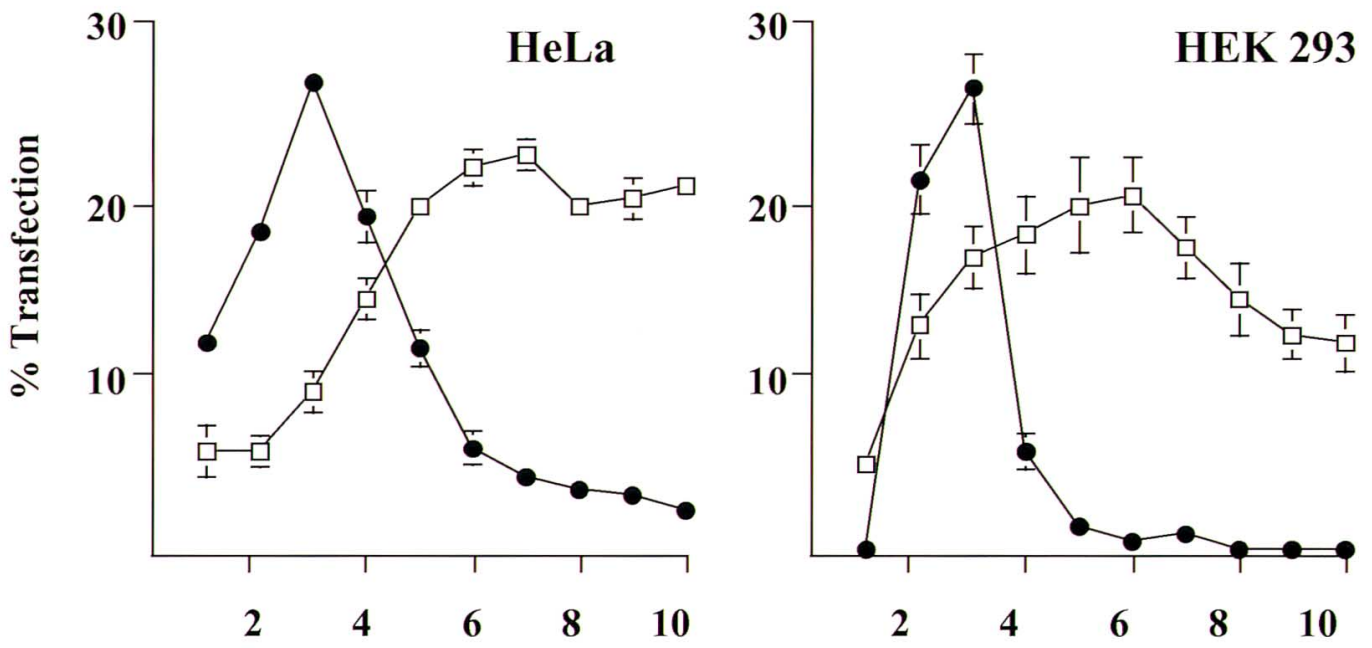

Outer distance from target centre, $\mathrm{mm}$

Fig. 3. Performance of standard (Bio-Rad) and modified apparatus on cell culture targets. (a) Representative images of the target centres of HeLa cell cultures, viewed with Hoechst staining to reveal all nuclei (left panels) or EYFP to identify transfected cells (right panels). The loss of cells from the target centres is revealed on the Hoechst stain. In addition, surrounding apoptotic nuclei are evident by their dense, irregular appearance. Scale bar $=1 \mathrm{~mm}$. The standard apparatus (upper panels) destroyed cells in the target centre and produced limited transfection. At 50 psi the modification caused less cell loss (middle panels), with little evidence of apoptosis but it did yield extensive transfection. At 75 psi extensive apoptosis was evident around the target centre (lower panels), although acute cell loss was limited, and transfection levels were again high when compared to the standard Gene Gun. (b) Quantitative analysis of transfection across HeLa and HEK293 cell cultures shot with standard (open squares, $175 \mathrm{psi}$ ) or modified (closed circles, $50 \mathrm{psi}$ ) apparatus held at $50 \mathrm{~mm}$ from the coverslips. Transfection frequency, determined as \% of Hoechst stained nuclei expressing EYFP is plotted radially with increasing distance from target centre. Data represent mean \pm SEM of three coverslips. The experiment was conducted independently three times with comparable results. 

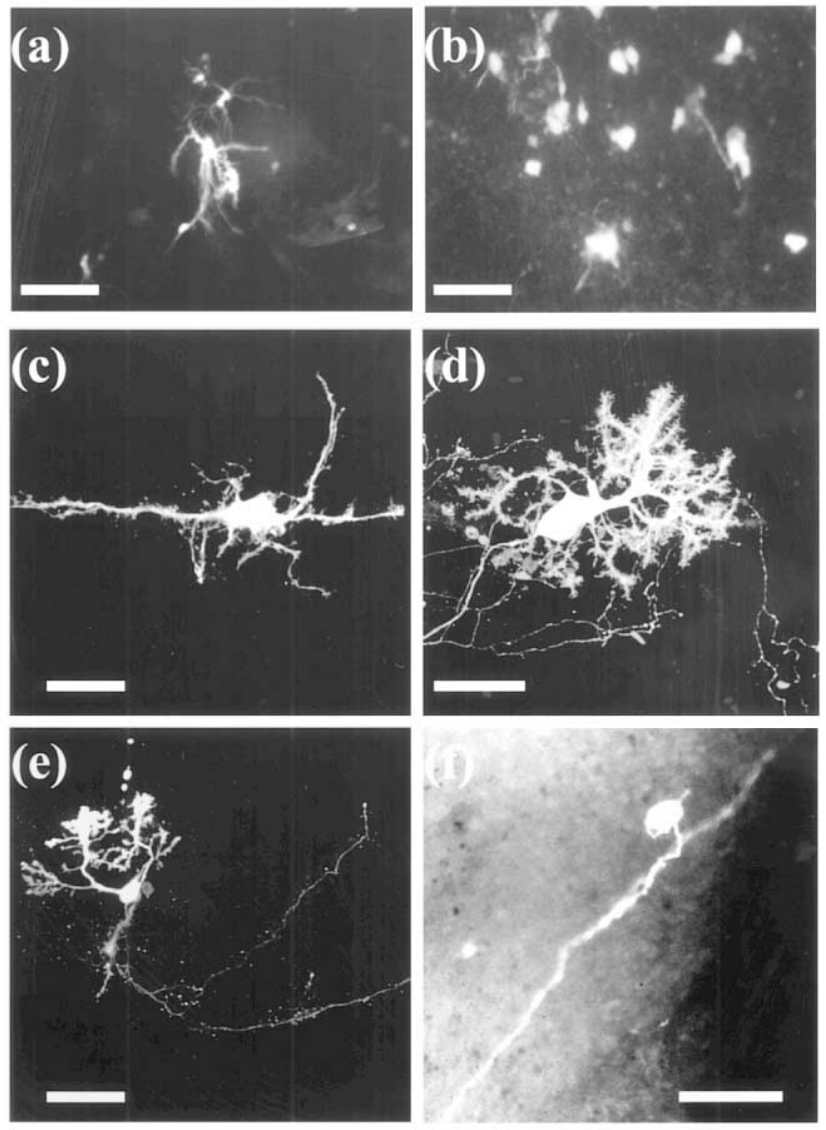

Fig. 4. Transfection of organotypic cultures by standard and modified Gene Gun apparatus. (a, b) Representative fields of view of cerebellar organotypic slices shot with CMV-EYFP bullets. The standard Gene Gun was shot with the spacer $10 \mathrm{~mm}$ from the tissue, whereas the end of the external barrel of the modified apparatus was held $50 \mathrm{~mm}$ from the target. Note the sparse number of transfected cells using the standard apparatus (a), whereas the modified Gene Gun (b) transfected significantly more cells in the target centre (defined as $100 \mu \mathrm{m}$ or more from the border of the tissue slice). Scale bar $=100 \mu \mathrm{m}$. (c, d, e) Representative neuronal cell profiles from cerebellar slices, viewed with confocal microscopy (40 or $60 \times$ ), after transfection with the modifed apparatus fired at 75 psi. These cells were located 174,189 and $184 \mu \mathrm{m}$ below the slice surface, respectively. Scale bars $=25,50$ or $100 \mu \mathrm{m}$, respectively. (f) Representative cell profile, viewed under epifluorescence, of pseudo-unipolar sensory afferent neuron from dorsal root ganglia shot with the modifed apparatus at 75 psi $(40 \times$, scale bar $=100 \mu \mathrm{m})$.

fired at $175 \mathrm{psi}$, and $111 \pm 22$ or $83 \pm 20 \mu \mathrm{m}$ for the modified apparatus fired at 75 or 50 psi, respectively $(n=12$ coverslips per determination). Together, these results confirm the restricted target area of the modified apparatus, and also demonstrate its effectiveness at low helium pressures, a major advantage when working with dispersed cell cultures because it reduces cell loss in the target centre.

The standard Bio-Rad apparatus used at 175 psi was able to transfect cells in organotypic slices of mouse cerebellum (Fig. 4a). However, these cells were distributed predominantly around the periphery of the slice and were most probably astrocytes migrating out from the tissue in a monolayer. Few cells $(23.8 \pm 13.4$ cells/slice, mean \pm SEM, $n=30$ slices) were evident in the centre of the slice (defined as more than $100 \mu \mathrm{m}$ from the tissue border). Confocal microscopy (Bio-Rad 'Radiance 2000' scanner operated with Nikon Eclipse TE300 microscope) revealed that the cells transfected with the standard apparatus were located no deeper than $150 \mu \mathrm{m}$ below the slice surface. In contrast, the modified apparatus transfected significantly more cells in the centre of the slice than did the standard apparatus $(70.0 \pm 17.8$, cells/slice $n=30$ slices, $P<0.01$ by Student's $t$-test) (Fig. 4b). Moreover, these cells were located at depths of up to $280 \mu \mathrm{m}$ in the ca. $300 \mu \mathrm{m}$ deep slices. Representative cell profiles located at depths of approximately $185 \mu \mathrm{m}$, as determined by confocal microscopy, are presented in Fig. 4. The cells transfected by the modified apparatus exhibited a range of morphologies, including definitive Purkinje neurons and other neuronal profiles, as well as astrocytic profiles (Fig. 4c, d, e).

To optimise the procedure further, Tefzel cartridges were loaded with increasingly more dilute gold suspension. A 1 in 2 dilution had no significant effect on the frequency of transfection (mean $\pm \mathrm{SEM}, n=20$ slices per group: standard loading, $96.6 \pm 5.6$ cells/slice; 1 in 2 dilution, $92.2 \pm 10.7$ cells/slice). However, a 1 in 4 dilution attenuated the level of transfection significantly $(39.0 \pm 5.5$ cells/slice, $P<0.01$ vs. standard loading).

Finally DRG explant cultures were subject to transfection, as a further assessment of the performance of the modified apparatus on a three-dimensionally complex target. When shot at 100,75 or 50 psi, the standard apparatus did not produce any transfected cells in the DRG tissues ( $n=3$ explants for all three pressures). The standard apparatus used at 175 psi produced relatively few transfected cells $(3.5 \pm 0.8$ cells/explant, mean \pm SEM, 21 explants tested). In contrast to the results from dispersed cell culture (HeLa and HEK293, see above) the modified apparatus was not effective when used at $50 \mathrm{psi}(n=6$ explants). This may reflect a protective action of any connective tissue capsule surrounding the DRG. The modified Gene Gun was, however, highly effective when used at 75 psi, transfecting significantly more cells than the standard Gene Gun used at $175 \mathrm{psi}(14.4 \pm 2.0$ cells/slice, $n=21$ slices, $t$-test comparison $P<0.01)$. A large proportion of the cells transfected with the modified apparatus $(66 \pm 6.3 \%$ in six DRG cultures) were clearly pseudo-unipolar primary afferent neurons (Fig. 4f). These results confirm the ability of the modified Gene Gun, used at relatively low gas pressures, to propel gold particles deeper into the target tissue. They also demonstrate that the particles remain effective vectors for plasmid transfection of neurons. 


\section{Conclusion}

The hand-held Bio-Rad Gene Gun is a convenient and effective means of transfecting cultured cells, including neurons (Yoshida et al., 1997; Wellmann et al., 1999). It offers clear advantages of flexibility of use when compared with the vacuum chamber format reported for neuronal transfection by earlier studies. Our modifications to the apparatus enhance its accuracy by limiting the spread of gold microcarriers, and also increase its tissue penetration whilst allowing it to operate at a reduced helium pressure. These enhancements make the hand-held Gene Gun more effective for use both on cell cultures, where tissue damage in the centre of the target was reduced, and also on organotypic cultures which require deeper penetration for effective transfection.

The behaviour of the standard hand-held Gene Gun when fired at inert targets was very similar to that reported previously by Yoshida et al. (1997). There was very little expulsion of radiolabelled DNA when the gun was fired at 100 psi. When shot at 200 psi and $4 \mathrm{~cm}$ from the target surface, the radiolabel tracer revealed a target area of $1.2 \mathrm{~cm}$ (cf. $1.5 \mathrm{~cm}$ in our study). Moreover, penetration into agar was in the region of $400 \mu \mathrm{m}$ when shot at $250 \mathrm{psi}$ (cf. $100 \mu \mathrm{m}$ in our study).

When tested at 100 psi or below the standard apparatus was not effective for transfection. This is consistent with some, but not all, reports. For example Yoshida et al. (1997) used it at 150 psi to transfect COS cells, and 250 psi to transfect liver in vivo, whilst Zhang and Selzer (2001) reported 175 psi as optimal for transfection of lamprey brain tissue. Nevertheless, other studies have reported effective transfection using this apparatus at 100 psi, (see Wellmann et al., 1999), although it has been suggested that repeated firings be used at $100 \mathrm{psi}$ to maximise transfection (Gan et al., 2000). The discrepancy in the optimum pressures noted between laboratories may be related to the coating of Tefzel tubing with gold/DNA suspension. In our preliminary studies, difficulties were encountered in achieving adequate surface coating of the tubing with gold particles. The suppliers ultimately attributed this to a change in the plasticizer used in manufacture of the tubing. The coating difficulty was overcome by irradiation of the Tefzel tubing, originally adopted as a sterilising procedure (Manufacturer's communication). Nevertheless, the new specification of the tubing altered its characteristics, in particular the ease with which gold particles could be coated on to it, and subsequently stripped off by the helium jet. As a result, the current study needed to use pressures higher than those reported as optimal in earlier work (Wellmann et al., 1999) to displace gold particles from the cartridge and achieve transfection. In our hands, firing the standard Gene Gun at 100 psi or below markedly reduced the number of gold particles stripped from the cartridge, approximately halved the penetration of any gold that was released, and did not lead to appreciable transfection of either dispersed cells or organotypic tissues.

Even though the standard Gene Gun had to be used at a higher pressure (175 psi), the profile of transfection of HeLa and HEK293 cells achieved in the current study was comparable to that reported by Wellmann et al. (1999) for dispersed neuronal cultures. First, there was a marked 'dead-zone' in the centre, attributed to gas impact and a heavy gold particle loading. A similar dead-zone of $1.1 \mathrm{~cm}$ diameter was also reported by Yoshida et al. (1997) working with COS cells transfected using the standard gun fired at 150 psi. In our study and that of Wellmann et al. (1999) there was a radial increase in transfection frequency with increasing distance from the centre. This peaked in the range of $5-8 \mathrm{~mm}$ from the target centre, and then tended to fall away again. The current study achieved a maximum of ca. $22 \%$ transfection using cell lines, whereas transfection of primary neuronal cultures peaked at $10 \%$. The difference may well reflect the relative robustness of the cells rather than any systematic difference between the apparatus used. Overall, therefore, these data demonstrate that regardless of the higher pressure used, the ultimate performance of the standard Bio-Rad Gene Gun on cell cultures was comparable between our own and previous studies. It is against this standard that the performance of the modified apparatus is to be judged. Of particular note is the effectiveness of the modified apparatus at pressures well below the range reported for use with the standard apparatus, and its more focussed area of transfection in cell cultures, peaking at ca. $25 \%$ within $3 \mathrm{~mm}$ of the target centre.

Further enhancements to performance of the modified Gene Gun may be envisaged. For example, optimisation of gold loading against gas pressure should now be possible with the modified apparatus, and this should reduce the potential for tissue damage even further and make the procedure more economic. In our own and other studies (see Wellmann et al., 1999) the use of a $70 \mu \mathrm{m}$ mesh between Gene Gun and target was essential for effective transfection. The use of a finer mesh with $3 \mu \mathrm{m}$ pore size to cover the end of the accelerator channel has been recommended (Gan et al., 2000), and this may help to reduce tissue damage further. Finally, the greater penetration revealed on inert targets and on cultured tissue in vitro remains to be tested in vivo. To date, the hand-held Gene Gun has been useful for 'in situ' transfection of cells in mammalian skin (Johnston and Tang, 1993) but has limited effectiveness for transfection of deeper tissues (Hui et al., 1994). The modification described here offers the prospect of more extensive in vivo transfection of subepidermal tissues, and possibly deeper brain tissues when exposed in situ. 


\section{Acknowledgements}

We thank Steve Scotcher (MRC-LMB Workshop) for construction of the modifications to the Bio-Rad Gene Gun; Dr R.W. Colburn (University of Cambridge, Department of Anaesthesia) for assistance with preliminary studies involving dorsal root ganglion cultures, and Deborah O'Brien for her assistance in graphics. This study was supported by the Medical Research Council, UK and by a Wellcome Trust Senior Research Fellowship in Basic Biomedical Sciences to SCRL.

\section{References}

Arnold D, Feng L, Kim J, Heintz N. A strategy for the analysis of gene expression during neural development. Proc Natl Acad Sci 1994;91:9970-4.

Arnold DB, Heintz N. A calcium responsive element that regulates expression of two calcium binding proteins in Purkinje cells. Proc Natl Acad Sci 1997;94:8842-7.

Biewenga JE, Destree OH, Schrama LH. Plasmid-mediated gene transfer in neurons using the biolistics technique. J Neurosci Methods 1997;71:67-75.

Boynton JE, Gillham NW. Chloroplast transformation in Chlamydomonas. Meth Enzymol 1993;217:510-36.

Boynton JE, Gillham NW. Genetics and transformation of mitochondria in the green alga Chlamydomonas. Meth Enzymol 1996;264:279-96.

Gan W-B, Grutzendler J, Wong WT, Wong ROL, Lichtman JW. Multicolor 'diolistic' labeling of the nervous system using lipophilic dye combinations. Neuron 2000;27:219-25.

Hui KM, Sabapathy TK, Oei AA, Chia TF. Generation of allo-reactive cytotoxic $\mathrm{T}$ lymphocytes by particle bombardment-mediated gene transfer. J Immunol Methods 1994;171:147-55.

Jiao S, Cheng L, Wolff JA, Yang NS. Particle bombardment-mediated gene transfer and expression in rat brain tissues. Biotechnology 1993;11:497-502.

Johnston SA, Tang DC. In: Setlow JK, editor. The Use of Microparticle Injection to Introduce Genes into Animal Cells in Vitro and in Vivo in Genetic Engineering. New York: Plenum Press, 1993:225-36.
Katz LC, Lo DC, McAllister K. Neuronal transfection in brain slices using particle-mediated gene transfer. Neuron 1994;13:1263-8.

Klein TM, Wolf ED, Wu R, Sanford JC. High velocity microprojectiles for delivering nucleic acids into living cells. Nature 1987;327:70-3.

Klein TM, Fromm M, Weissinger A, Tomes D, Schaaf S, Sletten M, Sanford JC. Transfer of foreign genes into intact maize cells with high-velocity microprojectiles. Proc Natl Acad Sci 1988;85:43059.

Lo DC, McAllister AK, Katz LC. Neuronal transfection in brain slices using particle-mediated gene transfer. Neuron 1994;13:1263-8.

McAllister AK, Katz LC, Lo DC. Opposing roles for endogenous BDNF and NT-3 in regulating cortical dendritic growth. Neuron 1997; 18:767-78.

Murphy RC, Messer A. Gene transfer methods for CNS organotypic cultures: a comparison of three nonviral methods. Mol Ther 2001;3:113-21.

Sanford JC, Smith FD, Russell JA. Optimizing the biolistic process for different biological applications. Meth Enzymol 1993;217:483-509.

Tao X, Finkbeiner S, Arnold DB, Shaywitz AJ. Greenberg ME. $\mathrm{Ca} 2 \pm$ influx regulates BDNF transcription by a CREB family transcription factor-dependent mechanism. Neuron 1998;20:70926.

Thomas A, Kim DS, Fields RL, Chin H, Gainer H. Quantitative analysis of gene expression in organotypic slice-explant cultures by particle-mediated gene transfer. J Neurosci Methods 1998;84:181-91.

Usachev YM, Khammanivong A, Campbell C, Thayer SA. Particlemediated gene transfer to rat neurons in primary culture. Pflugers Arch 2000;439:730-8.

Wellmann H, Kaltschmidt B, Kaltschmidt C. Optimized protocol for biolistic transfection of brain slices and dissociated cultured neurons with a hand-held Gene Gun. J Neurosci Methods 1999;92:55-64.

Yoshida Y, Kobayashi E, Endo H, Hamamoto T, Yamanaka T, Fujimura A, Kagawa Y. Introduction of DNA into rat liver with a hand held Gene Gun: distribution of the expressed enzyme, [22 P]DNA, and $\mathrm{Ca}^{2 \pm}$ flux. Biochem Biophys Res Comm 1997;234:695-700.

Zelenin AV, Titomirov AV, Kolesnikov VA. Genetic transformation of mouse cultured cells with the help of high-velocity mechanical DNA injection. FEBS Lett 1989;244:65-7.

Zhang G, Selzer ME. In vivo transfection of lamprey brain neurons by Gene Gun delivery of DNA. Exp Neurol 2001;167:304-11. 